\title{
Pilot study of expanded carrier screening for 11 recessive diseases in China: results from 10,476 ethnically diverse couples
}

\author{
Sumin Zhao ${ }^{1,2} \cdot$ Jiale Xiang ${ }^{3}{ }^{3}$ Chunna Fan ${ }^{1,2} \cdot$ Asan $^{1,2} \cdot$ Xuan Shang $^{4} \cdot$ Xinhua Zhang $^{5} \cdot$ Yan Chen $^{6}$. \\ Baosheng Zhu ${ }^{7}$. Wangwei Cai ${ }^{8}$. Shaoke Chen ${ }^{9} \cdot$ Ren Cai ${ }^{10}$ - Xiaoling Guo ${ }^{11}$. Chonglin Zhang ${ }^{12}$. Yuqiu Zhou ${ }^{13}$.

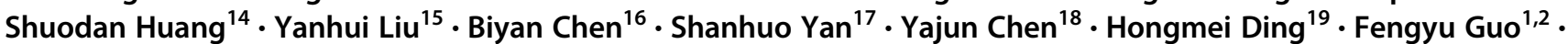 \\ Yaoshen Wang $\mathbb{1}^{1,2} \cdot$ Wenwei Zhong ${ }^{1,2} \cdot$ Yaping Zhu ${ }^{1,2} \cdot$ Yaling Wang ${ }^{1,2} \cdot$ Chao Chen $^{1,2} \cdot$ Yun $\mathrm{Li}^{20} \cdot$ Hui Huang ${ }^{3}$. \\ Mao $\mathrm{Mao}^{3} \cdot \mathrm{Ye} \mathrm{Yin}^{3} \cdot$ Jian Wang ${ }^{21,22} \cdot$ Huanming Yang ${ }^{21,22} \cdot$ Xiangmin $\mathrm{Xu}^{4} \cdot$ Jun Sun ${ }^{1,2} \cdot$ Zhiyu Peng $\mathbb{D}^{3,23}$
}

Received: 15 February 2018 / Revised: 20 July 2018 / Accepted: 9 August 2018 / Published online: 1 October 2018

(c) The Author(s) 2018. This article is published with open access

\begin{abstract}
Expanded carrier screening (ECS) has been demonstrated to increase the detection rate of carriers compared with traditional tests. The aim of this study was to assess the potential value of ECS for clinical application in Southern China, a region with high prevalence of thalassemia and with diverse ethnic groups, and to provide a reference for future implementations in areas with similar population characteristics. A total of 10,476 prenatal/preconception couples from 34 self-reported ethnic groups were simultaneously tested and analyzed anonymously for 11 Mendelian disorders using targeted next-generation sequencing. Overall, $27.49 \%$ of individuals without self-reported family history of disorders were found to be carriers of at least 1 of the 11 conditions, and the carrier frequency varied greatly between ethnic groups, ranging from $4.15 \%$ to $81.35 \%$. Furthermore, 255 couples $(2.43 \%)$ were identified as carrier couples at an elevated risk having an affected baby, sixty-five of which would not have been identified through the existing screening strategy, which only detects thalassemia. The modeled risk of fetuses being affected by any of the selected disorders was 531 per 100,000 (95\% CI, 497-567 per 100,000). Our data demonstrate the feasibility of ECS, and provide evidence that ECS is a promising alternative to traditional one-condition screening strategies. The lessons learned from this experience should be applicable for other countries or regions with diverse ethnic groups.
\end{abstract}

\section{Introduction}

Prenatal/preconception carrier screening, which aims to identify individuals or couples at risk of passing on recessive monogenic disorders to their offspring, has been accepted as an effective early intervention strategy to reduce the prevalence of disability and disease in the newborn

These authors contributed equally: Sumin Zhao, Jiale Xiang.

Electronic supplementary material The online version of this article (https://doi.org/10.1038/s41431-018-0253-9) contains supplementary material, which is available to authorized users.

Jun Sun

sunjun@bgi.com

Zhiyu Peng

pengzhiyu@bgi.com

Extended author information available on the last page of the article. population. A carrier screening program for Tay-Sachs disease started in 1971 [1] and has become the prototype for carrier screening of recessive disorders.

Advances in sequencing technology and decreases in cost [2] have made expanded carrier screening (ECS) feasible and affordable. In 2011, after 14 years of cumulative experience in gene-by-gene carrier screening, screening tests were first expanded to simultaneously test for 448 Mendelian recessive diseases using next-generation sequencing (NGS) technology [3]. Since then, ECS has been implemented in several populations, and the power of NGS and expanded panels increases detection rates compared with traditional tests [4-7].

It is known that the carrier frequencies of Mendelian disorders differ considerably between ethnic groups [7, 8]. Scientific guidelines announced by the American College of Obstetricians and Gynecologists (ACOG), the American College of Medical Genetics and Genomics (ACMG), and other professional institutes dictate careful vetting of 
demographic characteristics, such as ethnic background, during carrier screening [9-12]. Knowing the frequency of variants in the population being tested facilitates pan-ethnic testing, but this is generally unrealistic to achieve due to costs and concerns of stigmatization. Moreover, geographical mobility is continuously increasing, also in China [13], increasing the possibility of mixed marriages and the complexity of genetic backgrounds.

China is a populous country with high incidence of birth defects [14], and has 56 ethnic groups (the Han and 55 ethnic minorities) $[15,16]$ which typically have their own distinctive culture and language. Several studies have elucidated some of the genetic variation in these groups [17-19]. These population characteristics provide a good opportunity to explore the performance of ECS in area with diversified ethnicities.

Here, we report the first design and validation of an ECS panel covering 11 Mendelian disorders using targeted NGS in China. The testing of the ECS in 10,476 ethnically diverse couples simultaneously supports its feasibility and provides evidence that ECS is useful in a region with diverse ethnic groups. We also identified several groups at considerable risk of passing genetic variants to their offspring, indicating a potential need for expanded carrier screening.

\section{Materials and methods}

\section{Study population}

The samples were categorized into two groups. The first group included 2238 patients or carriers with known genetic variants and was used for the validation of the ECS panel. The second group consisted of samples from 10,527 couples without a self-identified family history of inherited disorders, who were selected from five provinces in southern China for a hemoglobinopathies research project [20]. The pre-test genetic counseling for the hemoglobinopathies project was free to access. Basic genetic knowledge, such as inheritance patterns was spread through posters in hospitals while the hemoglobinopathies research project was underway. These couples were informed that the remaining samples might be used for other research purposes after anonymization and de-identification. Informed consent was required for all individuals.

Fifty-one of these couples were excluded from further analysis because either or both of the partners' DNA failed the sample quality control. Members of a couple underwent the test simultaneously and were required to report their ethnic background. Individuals in China identify as part of an ethnic group only if at least one of their parents belongs to the group. As this study was performed 1 year after sample collection, the testing results were not informed to individuals. The Institutional Review Board (IRB) of BGI approved the study.

\section{Disease selection and panel design}

The selection of monogenic diseases followed the criteria recommended by the ACMG, including considerations regarding prenatal diagnosis and a validated clinical association between mutation(s) and disease severity [12]. Eleven recessive disorders were included, namely $\alpha$-thalassemia (OMIM 604131); $\beta$-thalassemia (OMIM 613985); Phenylalanine hydroxylase deficiency (PAH deficiency; OMIM 261600); Wilson disease (WD; OMIM 277900); GJB2related DFNB1 nonsyndromic hearing loss and deafness (DFNB1; OMIM 220290); Deafness, autosomal recessive 4, with enlarged vestibular aqueduct (DFNB4; OMIM 600791)/ Pendred syndrome (PDS; OMIM 274600); Pompe disease (PD; OMIM 232300); Autosomal recessive polycystic kidney disease (ARPKD; OMIM 263200); Hyperphenylalaninemia, BH4-deficient, A (HPABH4A; OMIM 261640); Galactosemia (OMIM 230400); and Dystrophinopathies (Duchenne muscular dystrophy; OMIM 310200 \& Becker muscular dystrophy; OMIM 300376). The severity of these diseases was classified as moderate, severe or profound [21] (Supplementary Table 1).

Twelve genes were selected for the design of a customized capture array (NimbleGen, Roche) to capture the target regions of interest, including all exons along with 60-bp flanking intronic sequences and certain wellcharacterized intron sequences. For thalassemia, in addition to the above-mentioned regions, known deleterious copy number variants (CNVs) listed in HbVar (http:// globin.cse.psu.edu/hbvar/) outside exons and single nucleotide variants (SNVs)/insertion and deletion variants (indels) were included. Additionally, 21 autosomal SNPs and 2 genes (PS4Y1 and AMELY) on the Y-chromosome were included for internal quality assurance. The total size of targeted regions was $386,605 \mathrm{bp}$.

\section{NGS and data analysis}

Genomic DNA extraction was carried out with a MagPure Buffy Coat DNA Midi KF Kit (Magen, China) from peripheral blood. The DNA was then sequenced on a HiSeq 2000 or 4000 (Illumina, San Diego, USA) in 100-bp pairedend reads. The capture of targeted regions, enrichment, elution, and data analysis was performed according to a previously published protocol [20]. The reference genome sequence used was hg19.

Classification and interpretation of variants were performed according to the standards and guidelines recommended by ACMG and published in the literature [22-24]. The classification criteria used to support pathogenicity of 
the variants are listed in Supplementary Table 2. A variant database was constructed as previously described [25]. Our final database included a total of 2319 variants, including 1310 SNVs, 432 indels and 577 CNVs. Individuals were identified as carriers if they were positive either for variants in our database or for loss-of-function (LOF) variants not listed in our database that have an allele frequency lower than 0.001 in the 1000 Genomes Project (www. 1000genomes.org/) and dbSNP (www.ncbi.nlm.nih.gov/ $\mathrm{SNP} /$ ). If both the male and the female were carriers of a same autosomal recessive disorder or the female was the carrier of an X-linked recessive disorder, they were identified as a carrier couple at increased risk having an affected baby.

\section{Results}

\section{Validation study}

We first evaluated the performance of the NGS-based screening assay. The Yan Huang genome sample [26] was utilized as reference material to assess the sequencing depth needed for detecting variants of target regions. When the sequencing depth was greater than 100-fold, the sensitivity, specificity, accuracy, positive predictive value, and negative predictive value of SNVs on the targeted regions $(\geq 20$ depth) reached 99.9\% (Supplementary Figure 1). Coverage of the targeted regions ( $\geq 20$ depth) was also above $99 \%$ (Supplementary Figure 2). We next tested the reproducibility of the assay and detected no inconsistent variants between intra- and inter-run replicates, giving a concordance rate of $100 \%$. The consistency of the same regions in $\geq 20$-fold coverage was over $99.8 \%$ (Supplementary Table 3-4).

We also simulated 100 genomes containing 1743 deleterious variants to appraise the performance of the assay. When the mean sequencing depth was $\geq 100$-fold, the accuracy, specificity, and sensitivity of variant detection were all over 99.9\% (Supplementary Table 5). Finally, we analyzed 2238 samples with 4322 known variants to validate the ECS panel (Supplementary Table 6). Overall, 4321 of the 4322 variants were detected. The undetected variant was the partial deletion $(30 \mathrm{bp}$ ) of exon 52 in the $D M D$ gene, which was removed during quality filtration due to the small size of deletion. In general, on the basis of the validation results, a mean sequencing depth of $\geq 100$-fold was aimed for in the study of 10,476 couples.

\section{Population demographics}

The screening population included 10,476 couples $(20,952$ individuals) with an average age of 29.23 years and a median age of 28 years. Approximately $96.71 \%(10,131 /$ $10,476)$ of the couples included ethnic information for both partners, with $2.68 \%(n=281)$ having ethnic information for only one partner and $0.61 \%(n=64)$ not identified to any ethnicity. In total, 34 self-reported Chinese ethnic groups were included. The largest population was the Han ethnicity $(n=14,233)$, comprising $67.93 \%$, with minorities accounting for $30.12 \%(n=6310)$ and $1.95 \%(n=409)$ of individuals without a reported ethnic group (Supplementary Table 7). The top ten minority groups in the south of China were all represented in the study, namely the Zhuang, Hui, Miao, Yi, Tujia, Dong, Buyei, Yao, Bai, and Hani [16]. Fourteen ethnic groups which had over one hundred representatives in this study were included for further analysis and discussion.

\section{Disease carrier frequencies}

Data about the variants identified in this study has been deposited in Genome Variation Map at http://bigd.big.ac.cn/ gvm/getProjectDetail?project=GVM000025. The carrier frequencies of the 11 conditions are presented in Table 1. Of the 20,952 individuals screened, $27.49 \%(n=5707)$ were found to be carriers of at least one condition. The most common disease was $\alpha$-thalassemia, with a rate of $15.12 \%$, extraordinarily high compared with other diseases detected in this study. This was four times greater than PAH deficiency $(n=753,3.59 \%)$ and three times greater than

Table 1 Carrier frequencies of 11 recessive diseases in cohort of 10,476 couples

\begin{tabular}{|c|c|c|c|}
\hline \multirow[t]{2}{*}{ Disease } & \multicolumn{3}{|c|}{ Carrier frequency } \\
\hline & $\mathrm{N}$ & $\%^{\mathrm{a}}$ & 1 in \\
\hline All & 5707 & 27.49 & 4 \\
\hline$\alpha$-thalassemia & 3167 & 15.12 & 7 \\
\hline$\beta$-thalassemia & 995 & 4.75 & 21 \\
\hline Phenylalanine hydroxylase deficiency & 753 & 3.59 & 28 \\
\hline Wilson disease & 410 & 1.96 & 51 \\
\hline DFNB1 & 347 & 1.66 & 60 \\
\hline DFNB4/PDS & 333 & 1.59 & 63 \\
\hline Pompe disease & 158 & 0.75 & 133 \\
\hline ARPKD & 118 & 0.56 & 178 \\
\hline HPABH4A & 52 & 0.25 & 403 \\
\hline Galactosemia & 26 & 0.12 & 806 \\
\hline Dystrophinopathies $^{\mathrm{b}}$ & 13 & 0.12 & 806 \\
\hline
\end{tabular}

DFNB1 GJB2-related DFNB 1 nonsyndromic hearing loss and deafness, DFNB4 deafness, autosomal recessive 4, with enlarged vestibular aqueduct, $P D S$ Pendred syndrome, ARPKD autosomal recessive polycystic kidney disease, $H P A B H 4 A$ hyperphenylalaninemia, BH4-deficient, A

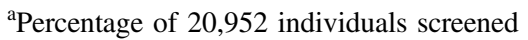

${ }^{\mathrm{b}}$ Carrier frequency in female 
Table 2 Carrier frequencies categorized by ethnicity

\begin{tabular}{|c|c|c|c|c|c|c|c|c|c|c|}
\hline \multirow[t]{2}{*}{ Ethnicity } & \multirow[t]{2}{*}{ Number of screened } & \multicolumn{3}{|c|}{$\begin{array}{l}\text { Total carrier } \\
\text { frequency }\end{array}$} & \multicolumn{3}{|c|}{$\begin{array}{l}\text { Carriers for one } \\
\text { disease }\end{array}$} & \multicolumn{3}{|c|}{$\begin{array}{l}\text { Carriers for two or } \\
\text { more diseases }\end{array}$} \\
\hline & & $N$ & $\%$ & 1 in & $N$ & $\%$ & 1 in & $N$ & $\%$ & 1 in \\
\hline All & 20,952 & 5707 & 27.49 & 3.6 & 5083 & 24.44 & 4.1 & 624 & 3.05 & 32.7 \\
\hline $\mathrm{Li}$ & 563 & 458 & 81.35 & 1.2 & 398 & 70.69 & 1.4 & 60 & 10.66 & 9.4 \\
\hline Dai & 226 & 90 & 39.82 & 2.5 & 76 & 33.63 & 3.0 & 14 & 6.19 & 16.1 \\
\hline Zhuang & 1989 & 710 & 35.70 & 2.8 & 619 & 31.12 & 3.2 & 91 & 4.58 & 21.9 \\
\hline Yao & 144 & 42 & 29.17 & 3.4 & 33 & 22.92 & 4.4 & 9 & 6.25 & 16.0 \\
\hline Miao & 733 & 199 & 27.15 & 3.7 & 171 & 23.33 & 4.3 & 28 & 3.82 & 26.2 \\
\hline Buyei & 333 & 87 & 26.13 & 3.8 & 78 & 23.42 & 4.3 & 9 & 2.70 & 37.0 \\
\hline Han & 14,233 & 3598 & 25.28 & 4.0 & 3224 & 22.65 & 4.4 & 374 & 2.63 & 38.1 \\
\hline Dong & 643 & 154 & 23.95 & 4.2 & 140 & 21.77 & 4.6 & 14 & 2.18 & 45.9 \\
\hline Tujia & 163 & 32 & 19.63 & 5.1 & 31 & 19.02 & 5.3 & 1 & 0.61 & 163.0 \\
\hline Sui & 199 & 39 & 19.60 & 5.1 & 37 & 18.59 & 5.4 & 2 & 1.01 & 99.5 \\
\hline Bai & 280 & 54 & 19.29 & 5.2 & 51 & 18.21 & 5.5 & 3 & 1.43 & 70.0 \\
\hline Yi & 452 & 68 & 15.04 & 6.6 & 64 & 14.16 & 7.1 & 4 & 0.88 & 113.0 \\
\hline $\mathrm{Va}$ & 131 & 15 & 11.45 & 8.7 & 15 & 11.45 & 8.7 & - & - & - \\
\hline Hani & 241 & 10 & 4.15 & 24.1 & 10 & 4.15 & 24.1 & - & - & - \\
\hline Others & 213 & 39 & 18.31 & 5.5 & 32 & 15.02 & 6.7 & 7 & 3.29 & 30.4 \\
\hline Unknown & 409 & 112 & 27.38 & 3.7 & 104 & 25.43 & 3.9 & 8 & 1.96 & 51.1 \\
\hline
\end{tabular}

Others included ethnicities from Hui, Lahu, Gelao, Blang, Mulao, Manchu, Lisu, Mongol, Jino, Tibetan, Maonan, Naxi, Chuangqing, De'ang, Pumi, Achang, Korean, Gin, Jingpo, San.

— indicates "not detected" $\beta$-thalassemia $(n=995,4.75 \%)$. The carrier frequencies of five disorders are reported for the first time in the Chinese population: WD $(n=410,1.96 \%)$, ARPKD $(n=118$, $0.56 \%)$, HPABH4A $(n=52,0.25 \%)$, galactosemia $(n=26$, $0.12 \%$ ), and dystrophinopathies ( $n=13,0.12 \%$ of females).

\section{Carrier frequencies categorized by ethnicity}

The overall carrier frequencies varied substantially between ethnic groups, ranging from $4.15 \%$ of individuals of Hani ethnicity to $81.35 \%$ of individuals of Li ethnicity (Table 2). Three ethnic groups (the Dai, Zhuang, and Yao) demonstrated high positive rates for at least one disease on the ECS panel $(39.82 \%, 35.70 \%$, and $29.17 \%$, respectively). The Han ethnicity, which is the largest ethnic group in China, had an overall carrier frequency of $25.28 \%$.

Of the 5707 carriers, $~ 89.07 \%(n=5083)$ were positive for one disorder and $10.93 \%(n=624)$ for two or more disorders (Table 2). The Li ethnicity again ranked first for multiple-disease carriers $(n=60,10.66 \%)$. Individuals from the Yao and Dai ethnicities were more likely than other ethnicities to be multiple-disease carriers as well $(6.25 \%$ and $6.19 \%$, respectively). Conversely, no Hani and Va individuals carrying multiple disease-causing variants were identified with this ECS panel.

The data were stratified by the ethnicity and disease to explore the variability among ethnic groups (Table 3). It is apparent that the selected diseases are widely spread among most of the ethnic groups. Variation in thalassemia carrier frequency predominantly contributed to the variability. The $\mathrm{Li}$, Dai, and Zhuang had a carrier frequency of $78.33 \%$, $27.43 \%$, and $22.83 \%$ for $\alpha$-thalassemia and $9.59 \%, 11.06 \%$, and $8.60 \%$ for $\beta$-thalassemia. Moreover, $14.32 \%$ of individuals from the Miao ethnic group were heterozygous for PAH deficiency, which was almost four times the overall frequency in the screened population $(3.59 \%)$ and twice the carrier frequency among the Sui (7.04\%). Finally, the Buyei group was at a greater than average risk of carrying ARPKD ( $1.80 \%$ vs. $0.56 \%$ overall) and the Bai of carrying DFNB1 (3.93\% vs. $1.66 \%$ overall).

\section{Carrier couples}

Overall, 254 carrier couples $(2.42 \%)$ were heterozygous for one condition, and one carrier couple $(0.01 \%)$ was heterozygous for both PAH deficiency and $\beta$-thalassemia (Table 4). Unsurprisingly, $\alpha$-thalassemia was the top disease in carrier couples $(n=137,1.31 \%)$, followed by $\beta$ thalassemia $(n=53,0.51 \%)$ and PAH deficiency $(n=34$, $0.32 \%)$.

The carrier burdens of couples by ethnicity were analyzed and groups represented by fewer than 20 couples are not listed (Supplementary Table 8). Of the forty-five Dai couples, $11.11 \%(n=5)$ were carrier couples, four times 


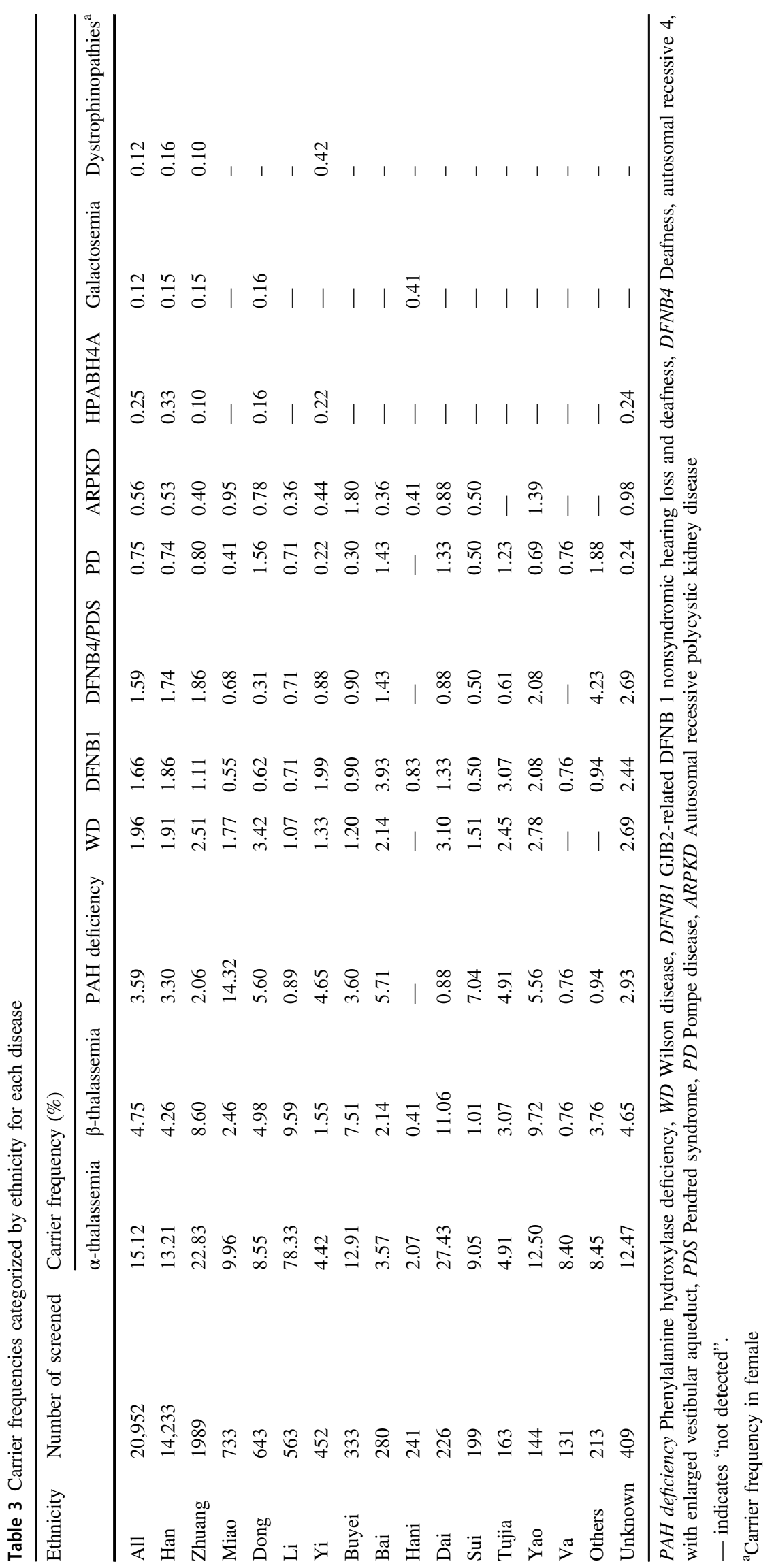


Table 4 Carrier couples by diseases

\begin{tabular}{|c|c|c|}
\hline Disease & $N$ & $\%^{\mathrm{a}}$ \\
\hline All & 255 & 2.43 \\
\hline Carrier couples for one disease & 254 & 2.42 \\
\hline$\alpha$-thalassemia & 137 & 1.31 \\
\hline$\alpha$-thalassemia, $\mathrm{Hb} \mathrm{H}$ & 102 & 0.97 \\
\hline$\alpha$-thalassemia, Hb Barts & 31 & 0.30 \\
\hline$\alpha$-thalassemia, $\mathrm{Hb} \mathrm{H}$ or $\mathrm{Hb}$ Barts & 4 & 0.04 \\
\hline$\beta$-thalassemia & 53 & 0.51 \\
\hline$\beta$-thalassemia intermedia & 28 & 0.27 \\
\hline$\beta$-thalassemia major & 25 & 0.24 \\
\hline PAH deficiency & 34 & 0.32 \\
\hline Dystrophinopathies ${ }^{\mathrm{b}}$ & 13 & 0.12 \\
\hline Wilson disease & 5 & 0.05 \\
\hline DFNB1 & 4 & 0.04 \\
\hline DFNB4/PDS & 4 & 0.04 \\
\hline Pompe disease & 2 & 0.02 \\
\hline ARPKD & 2 & 0.02 \\
\hline Carrier couple for two diseases & 1 & 0.01 \\
\hline $\begin{array}{l}\beta \text {-thalassemia intermedia \& Phenylalanine hydroxylase } \\
\text { deficiency }\end{array}$ & 1 & 0.01 \\
\hline
\end{tabular}

PAH deficiency Phenylalanine hydroxylase deficiency, DFNB1 GJB2related DFNB 1 nonsyndromic hearing loss and deafness, DFNB4 Deafness, autosomal recessive 4, with enlarged vestibular aqueduct, $P D S$ Pendred syndrome, ARPKD Autosomal recessive polycystic kidney disease, $N$ number of carrier couples

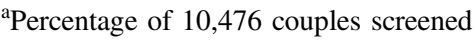

${ }^{\mathrm{b}}$ Carrier frequency in female

greater than the average (2.43\%). Of note, only $2.73 \%$ (5/ 183) of the Li couples were identified as carrier couples, despite a high carrier frequency in the Li ethnicity $(81.35 \%)$.

\section{Discussion}

\section{Selection of disorders}

The aims of carrier screening are to detect carriers of genetic disorders, to enhance reproductive autonomy, and to reduce the prevalence of disability and disease in the newborn population [27]. Therefore, one of the important criteria in our selection was disease severity. All the selected diseases were either profound or severe, with the exception of DFNB1 and DFNB4/PDS, which are considered moderate disorders. These two disorders were nevertheless included due to their high prevalence in the Chinese population [28], which was corroborated by this study. In addition, screening for hearing loss provides information which can facilitate earlier intervention to improve language development in affected children [29]. Although only 11 conditions were selected for this study, we have expanded our panel to a broader range including hundreds of conditions. A more comprehensive dataset will be published at a later date.

\section{Comparison to published carrier frequencies}

The carrier rates of thalassemia were higher in our study than published estimates for the Chinese population [30] based on the analysis of specific variants. This might be due to the adoption of targeted next-generation sequencing, which facilitated the identification of rare novel variants as well as reported variants that affect function [3, 6, 31]. Of the 376 identified deleterious or likely deleterious variants in our study, $82.98 \%(n=312)$ had already been reported in the literature but $17.02 \%(n=64)$ were novel, including nonsense, frameshift, deletion or duplication of exons, and splice site variants (Supplementary Table 9). The carrier frequencies of DFNB1 and DFNB4/PDS were similar to published estimates [28]. While Wilson disease has been investigated in a large Han population, the carrier frequency was not reported [32]. The remaining disorders have been subject to less investigation in the Chinese population, and study populations were either too small or limited to patients rather than including healthy individuals.

The difference in carrier frequencies compared with Western populations was striking. For example, the carrier frequency for dystrophinopathies generated in our study (1 in 806) was lower than that previously reported (1 in 433.7); likewise, 1 in 806 subjects was carrier of galactosemia, lower than the reported frequency of 1 in 100 [31]. Furthermore, the carrier rate for PAH deficiency (1 in 28) was higher compared to the Hispanic descent (1 in 163) and the southern Europeans ( 1 in 75) [8]. These differences may be attributed to differences in sample size, ethnic groups or variants detection range. These results also indicate that the disorders recommended for screening in Western societies might not be the correct targets in other populations.

\section{The need for expanding carrier screening}

Our data suggest a potential need to implement ECS in China for several reasons. First, around $27.49 \%$ of individuals were carriers for at least 1 of 11 selected diseases. Even when thalassemia is removed from our analysis, $10.02 \%$ of the individuals were still positive for at least one selected disorder. It is reasonable to believe that the frequency will increase when more diseases are included in the screening panel. Second, routine prenatal/preconception clinical screening for monogenic diseases (with the exception of thalassemia) is a novel practice in Chinese health care [17-20,33]. ECS would detect carrier couples affected by monogenic diseases other than thalassemia, which 
comprised $6.2 \%$ o $(65 / 10476)$ of our study population (Table 4). Third, one couple was found to be at risk of having a fetus with both $\beta$-thalassemia intermedia and PAH deficiency simultaneously. Traditional screening strategies would require multiple tests to identify two or more disorders. Fourth, according to calculations using a published method [7], the modeled risk of fetuses being affected by any of the selected disorders was 531 per 100,000 (95\% CI, 497-567 per 100,000), which translates into approximately 1 in 188 genetically affected births. This rate is comparable to the frequency of neonates affected by Down syndrome [34], for which free, government-subsidized screening is offered in routine antenatal tests in China.

We recommend that the screening strategy be universal rather than based on ethnicity. There are five reasons for this recommendation: (1) Thalassemia screening is currently recommended in southern China for all couples who are considering pregnancy or are already pregnant, regardless of their ethnicity, although the ethnic variability of thalassemia carrier frequency has been reported $[18,19]$. This serves as a good example which ECS should follow. (2) We have illustrated the variability of disease carrier frequencies in the different ethnic groups (Table 3), the complexity of which makes it unrealistic to develop screening panels for each of the 56 ethnic groups. (3) Information regarding variants in the population to be tested would be needed [12], but such data are barely available in the minority groups, especially those with a smaller population. (4) Ethnic-based screening may raise the risk of discrimination or stigmatization [9]. (5) Internal geographical mobility is continuously improving in China [13], which increases the possibility of mixed marriages and hinders ethnic identification. In our data, there were over 1127 (>11\%) couples of mixed ethnicity (Supplementary Table 8).

\section{Challenging for genetic counseling}

Undoubtedly, genetic counseling is an essential part of implementing carrier screening [35]. Our study provides valuable information about causative mutation(s) and mutation frequency in different ethnic groups which will greatly help clinical genetic counselors in risk evaluation. However, challenges remain, especially in the interpretation of variants of uncertain significance (VUS) generated from NGS. For instance, while the pathogenicity of VUS is controversial or the evidence for their classification is insufficient, they cannot be neglected because VUS have been reported as disease etiologies in some patients [36]. In our data, the paternal partner in one couple had the deleterious variant NM_000277.1: c.331C $>\mathrm{T}$ p. $\left(\operatorname{Arg} 111^{*}\right)$ in the $P A H$ gene, while the maternal partner had a VUS NM_000277.1: c.1144T>A p. (Phe382Ile) in the same gene. Determining the pathogenicity of p. (Phe382Ile) would be crucial for reproductive advice in this case. Furthermore, for females with an X-linked VUS detected, pathogenicity evaluation is a necessity to properly evaluate the implications for reproductive risk.

Furthermore, our data revealed that the variant spectrum of diseases in an ethnicity is uncertain and complex, which complicates genetic counseling. Specifically, around $2.73 \%$ of $\mathrm{Li}$ couples were found to be carrier couples (Supplementary Table 8), despite a much higher individual carrier rate of $81.35 \%$ among the $\mathrm{Li}$ (the $\alpha$-thalassemia carrier frequency was $78.33 \%$ ). This phenomenon was mainly due to the complicated variant spectrum of $\alpha$-thalassemia. In the Li ethnicity, the $-\alpha^{3.7}$ allele and $-\alpha^{4.2}$ alleles account for $72.95 \%$ of mutated alleles. Since the combination of $-\alpha^{3.7}$ and $-\alpha^{4.2}$ results in $\alpha$-thalassemia trait, a mild phenotype [37], couples with the two alleles were not classified as carrier couples.

\section{Clinical utility of ECS}

Our study revealed a wide range of carrier frequencies among the different ethnic groups in Southern China, highlighting the potential value of ECS. However, we only evaluated the clinical utility of ECS in terms of carrier and risk couple frequency distribution and test feasibility and validity. A proposed framework for the assessment of clinical utility of genetic testing should include the feasibility and validity of test, diagnostic thinking, therapeutic choices, patient outcomes and societal impacts [38]. Aspects other than test feasibility and validity and carrier and risk couple frequency need to be evaluated in future research for the full assessment of the clinical utility of ECS.

In summary, we report the first expanded carrier screening in China of 10,476 couples with diverse ethnicities. The data not only revealed the carrier frequencies of 11 monogenic disorders, but also suggested the potential utility of ECS in regions with diversified ethnic backgrounds. This experience should be applicable for other countries or regions with diverse ethnic groups. We note that most of the couples screened were from the Han ethnicity $(67.93 \%)$, and sample numbers of several ethnic groups were limited. However, this is consistent with the demographic characteristics of the Chinese population, in which the Han makes up the majority $(91.52 \%)$ according to the Sixth National Population Census in 2010 [16]. Nevertheless, we expect further examinations of these minority groups using a larger study population. Finally, we argue for the necessity of expanded carrier screening as a promising alternative and for genetic counseling, which is indispensable in the responsible implementation of expanded carrier screening. 
Acknowledgements We are grateful for the contributions of all the employees in Tianjin and Shenzhen Medical Laboratory, BGI. They provided strong support in the libraries construction, sequencing, data processing, etc. The study was supported by Tianjin Municipal Science and Technology Special Funds for Enterprise Development (No. 14ZXLJSY00320), Pearl River Nova Program of Guangzhou (No. 201506010100), Special Foundation for High-level Talents of Guangdong (2016TX03R171) and Natural Science Foundation of Guangdong Province (2017A030313673).

\section{Compliance with ethical standards}

Conflict of interest SZ, JX, CF, A, FG, YW, WZ, YZ, YW, CC, YL, $\mathrm{HH}, \mathrm{MM}, \mathrm{YY}, \mathrm{JW}, \mathrm{HY}$, JS, and ZP were employed at BGI at the time of submission. The remaining authors declare no conflict of interest.

Open Access This article is licensed under a Creative Commons Attribution 4.0 International License, which permits use, sharing, adaptation, distribution and reproduction in any medium or format, as long as you give appropriate credit to the original author(s) and the source, provide a link to the Creative Commons license, and indicate if changes were made. The images or other third party material in this article are included in the article's Creative Commons license, unless indicated otherwise in a credit line to the material. If material is not included in the article's Creative Commons license and your intended use is not permitted by statutory regulation or exceeds the permitted use, you will need to obtain permission directly from the copyright holder. To view a copy of this license, visit http://creativecommons. org/licenses/by/4.0/.

\section{References}

1. Kaback M, Lim-Steele J, Dabholkar D, Brown D, Levy N, Zeiger $\mathrm{K}$ : Tay-Sachs disease-carrier screening, prenatal diagnosis, and the molecular era. An international perspective, 1970 to 1993. The International TSD Data Collection Network. JAMA. 1993; 270: 2307-15.

2. National Human Genome Research Institute. The cost of sequencing a human genome, Vol 2016. Accessed at: https://www.genome.gov/sequencingcosts/.

3. Bell CJ, Dinwiddie DL, Miller NA, et al. Carrier testing for severe childhood recessive diseases by next-generation sequencing. Sci Transl Med. 2011;3:65ra64.

4. Beauchamp KA, Muzzey D, Wong KK, et al. Systematic design and comparison of expanded carrier screening panels. Genet Med. 2018;20:55-63.

5. Baskovich B, Hiraki S, Upadhyay K, et al. Expanded genetic screening panel for the Ashkenazi Jewish population. Genet Med. 2016;18:522-8

6. Martin J, Asan YiY, Rodríguez-Iglesias B, et al. Comprehensive carrier genetic test using next-generation deoxyribonucleic acid sequencing in infertile couples wishing to conceive through assisted reproductive technology. Fertil Steril. 2015;104:1286-93.

7. Haque IS, Lazarin GA, Kang HP, Evans EA, Goldberg JD, Wapner RJ. Modeled fetal risk of genetic diseases identified by expanded carrier screening. JAMA. 2016;316:734-42.

8. Lazarin GA, Haque IS, Nazareth S, et al. An empirical estimate of carrier frequencies for $400+$ causal Mendelian variants: results from an ethnically diverse clinical sample of 23,453 individuals. Genet Med. 2013;15:178-186.

9. Edwards JG, Feldman G, Goldberg J, et al. Expanded carrier screening in reproductive medicine-points to consider: a joint statement of the American College of Medical Genetics and Genomics, American College of Obstetricians and Gynecologists,
National Society of Genetic Counselors, Perinatal Quality Foundation, and Society for Maternal-Fetal Medicine. Obstet Gynecol. 2015;125:653-62.

10. American College of Obstetricians and Gynecologist. Committee Opinion No. 690: carrier screening in the age of genomic medicine. Obstet Gynecol. 2017;129:e35-40.

11. American College of Obstetricians and Gynecologist. Committee Opinion No. 691: carrier screening for genetic conditions. Obstet Gynecol. 2017;129:e41-55.

12. Grody WW, Thompson BH, Gregg AR, et al. ACMG position statement on prenatal/preconception expanded carrier screening. Genet Med. 2013;15:482-3.

13. Shen J. Increasing internal migration in China from 1985 to 2005 : Institutional versus economic drivers. Habitat Int. 2013;39:1-7.

14. Dai L, Zhu J, Liang J, Wang YP, Wang H, Mao M. Birth defects surveillance in China. World J Pediatr. 2011;7:302-10.

15. Zhang HG, Chen YF, Ding M, et al. Dermatoglyphics from all Chinese ethnic groups reveal geographic patterning. PLoS ONE. 2010;5:e8783.

16. National Bureau of Statistic of the People's Republic of China. The Sixth National Population Census, Vol 2010. Accessed at: http://www.stats.gov.cn/tjsj/pcsj/rkpc/6rp/indexch.htm.

17. Yao H, Chen X, Lin L, et al. The spectrum of alpha- and betathalassemia mutations of the $\mathrm{Li}$ people in Hainan Province of China. Blood Cells Mol Dis. 2014;53:16-20.

18. He J, Song W, Yang J, et al. Next-generation sequencing improves thalassemia carrier screening among premarital adults in a high prevalence population: the Dai nationality, China. Genet Med. 2017;19:1022-31.

19. Xiong F, Sun M, Zhang X, et al. Molecular epidemiological survey of haemoglobinopathies in the Guangxi Zhuang Autonomous Region of southern China. Clin Genet. 2010;78:139-48.

20. Shang X, Peng Z, Ye Y, et al. Rapid targeted next-generation sequencing platform for molecular screening and clinical genotyping in subjects with hemoglobinopathies. EBioMedicine. 2017;23:150-9.

21. Lazarin GA, Hawthorne F, Collins NS, Platt EA, Evans EA, Haque IS. Systematic classification of disease severity for evaluation of expanded carrier screening panels. PLoS ONE. 2014;9: e114391.

22. Richards CS, Bale S, Bellissimo DB, et al. ACMG recommendations for standards for interpretation and reporting of sequence variations: revisions 2007. Genet Med. 2008;10:294-300.

23. MacArthur DG, Manolio TA, Dimmock DP, et al. Guidelines for investigating causality of sequence variants in human disease. Nature. 2014;508:469-76.

24. Li MM, Datto M, Duncavage EJ, et al. Standards and guidelines for the interpretation and reporting of sequence variants in cancer: a joint consensus recommendation of the Association for Molecular Pathology, American Society of Clinical Oncology, and College of American Pathologists. J Mol Diagn. 2017;19:4-23.

25. Perreault-Micale C, Davie J, Breton B, Hallam S, Greger V. A rigorous approach for selection of optimal variant sets for carrier screening with demonstration of clinical utility. Mol Genet Genom Med. 2015;3:363-73.

26. Wang J, Wang W, Li R, et al. The diploid genome sequence of an Asian individual. Nature. 2008;456:60-5.

27. Kihlbom U. Ethical issues in preconception genetic carrier screening. Ups J Med Sci. 2016;121:295-8.

28. Zhang J, Wang P, Han B, et al. Newborn hearing concurrent genetic screening for hearing impairment-a clinical practice in 58,397 neonates in Tianjin, China. Int J Pediatr Otorhinolaryngol. 2013;77:1929-35.

29. Moeller MP. Early intervention and language development in children who are deaf and hard of hearing. Pediatrics. 2000;106: E43. 
30. Xu XM, Zhou YQ, Luo GX, et al. The prevalence and spectrum of alpha and beta thalassaemia in Guangdong Province: implications for the future health burden and population screening. J Clin Pathol. 2004;57:517-22.

31. Abuli A, Boada M, Rodriguez-Santiago B, et al. NGS-based assay for the identification of individuals carrying recessive genetic mutations in reproductive medicine. Hum Mutat. 2016; 37:516-23.

32. Cheng N, Wang $\mathrm{K}, \mathrm{Hu} \mathrm{W}$, et al. Wilson disease in the South chinese han population. Can J Neurol Sci. 2014;41:363-7.

33. Liao C, Mo QH, Li J, et al. Carrier screening for alpha- and betathalassemia in pregnancy: the results of an 11-year prospective program in Guangzhou Maternal and Neonatal hospital. Prenat Diagn. 2005;25:163-71.
34. Hook EB, Cross PK, Schreinemachers DM. Chromosomal abnormality rates at amniocentesis and in live-born infants. JAMA. 1983;249:2034-8.

35. Archibald AD, Smith MJ, Burgess T, et al. Reproductive genetic carrier screening for cystic fibrosis, fragile $\mathrm{X}$ syndrome, and spinal muscular atrophy in Australia: outcomes of 12,000 tests. Genet Med. 2018;20:513-23.

36. Yuan Y, You Y, Huang D, et al. Comprehensive molecular etiology analysis of nonsyndromic hearing impairment from typical areas in China. J Transl Med. 2009;7:79.

37. Galanello R, Cao A. Gene test review. Alpha-thalassemia. Genet Med. 2011;13:83-8.

38. Grosse SD, Khoury MJ. What is the clinical utility of genetic testing? Genet Med. 2006;8:448-50.

\section{Affiliations}

\section{Sumin Zhao ${ }^{1,2} \cdot$ Jiale Xiang $\mathbb{1}^{3} \cdot$ Chunna Fan $^{1,2} \cdot$ Asan $^{1,2} \cdot$ Xuan Shang $^{4} \cdot$ Xinhua Zhang $^{5} \cdot$ Yan Chen $^{6}$. Baosheng $\mathrm{Zhu}^{7} \cdot$ Wangwei $\mathrm{Cai}^{8} \cdot$ Shaoke Chen ${ }^{9} \cdot$ Ren Cai $^{10} \cdot$ Xiaoling Guo ${ }^{11}$. Chonglin Zhang ${ }^{12}$ - Yuqiu Zhou ${ }^{13}$. Shuodan Huang ${ }^{14}$. Yanhui Liu ${ }^{15}$. Biyan Chen ${ }^{16}$. Shanhuo Yan $^{17}$ - Yajun Chen ${ }^{18}$. Hongmei Ding ${ }^{19} \cdot$ Fengyu Guo $^{1,2}$.

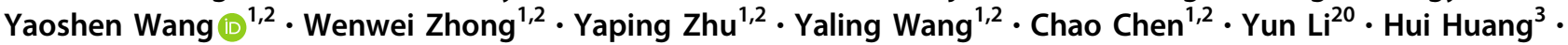 Mao $\mathrm{Mao}^{3} \cdot \mathrm{Ye} \mathrm{Yin}^{3} \cdot$ Jian Wang ${ }^{21,22} \cdot$ Huanming Yang ${ }^{21,22} \cdot$ Xiangmin $\mathrm{Xu}^{4} \cdot$ Jun Sun ${ }^{1,2} \cdot$ Zhiyu Peng $\circledast^{3,23}$}

1 Tianjin Medical Laboratory, BGI-Tianjin, BGI-Shenzhen, 300308 Tianjin, China

2 Binhai Genomics Institute, BGI-Tianjin, BGI-Shenzhen, 300308 Tianjin, China

3 BGI Genomics, BGI-Shenzhen, 518083 Shenzhen, China

4 Department of Medical Genetics, School of Basic Medical Sciences, Southern Medical University, 510515

Guangzhou, Guangdong, China

5 Department of Hematology, 303rd Hospital of the People's Liberation Army, Nanning, Guangxi, China

6 The Second Department of Pediatrics, Affiliated Hospital of Zunyi Medical College, Zunyi, Guizhou, China

7 Nation Health and Family Planning Commission Key Laboratory For Preconception and Health Birth in Western China, The First People's Hospital of Yunnan Province, Kunming University of Science and Technology, Kunming, China

8 Department of Biochemistry and Molecular Biology, Hainan Medical College, Haikou, Hainan, China

9 Department of Genetic and Metabolic Laboratory, Guangxi Zhuang Autonomous Region Women and Children Health Care Hospital, Nanning, Guangxi, China

10 Department of Medical Genetics, Liuzhou Municipal Maternity and Child Healthcare Hospital, Liuzhou, Guangxi, China

11 Maternity and Child Health Care Hospital of Foshan City, Foshan, Guangdong, China
12 Guilin Women and Children Health Care Hospital, Guilin, Guangxi, China

13 Department of Clinical Laboratory, Zhuhai Municipal Maternal and Child Healthcare Hospital, Zhuhai Institute of Medical Genetics, Zhuhai, Guangdong, China

14 Maternal and Child Health Hospital in Meizhou, Meizhou, Guangdong, China

15 Department of Prenatal Diagnosis Center, Dong Guan Maternal and Child Health Hospital, Dongguan, Guangdong, China

16 Baise Women and Children Care Hospital, Baise, Guangxi, China

17 Genetic Laboratory, Qinzhou Maternal and Child Health Hospital, Qingzhou, Guangxi, China

18 Women and Children's Health Hospital of Shaoguan, Shaoguan, Guangdong, China

19 Department of Gynecology and Obstetrics, The People's Hospital of Yunfu City, Yunfu, Guangdong, China

20 BGI Clinical Laboratories-Shenzhen, BGI-Shenzhen, 518083 Shenzhen, China

21 James D. Watson Institute of Genome Sciences, 310058 Hangzhou, China

22 BGI-Shenzhen, 518083 Shenzhen, China

23 BGI-Guangzhou Medical Laboratory, BGI-Shenzhen, 510006 Guangzhou, China 